\title{
Coexistence of inversion 16 in chronic myeloid leukaemia in blast crisis
}

\author{
Mark A. Catherwood ${ }^{1} \cdot$ Peter McGrattan $^{2} \cdot$ S. Lawless $^{1} \cdot$ C. McConville ${ }^{1} \cdot$ N. Robson ${ }^{1}$ • \\ B. Lundy ${ }^{1}$ - M. Humphreys ${ }^{2}$ - S. Soverini ${ }^{3}$ - K. I. Mills ${ }^{4}$ - M. F. McMullin ${ }^{1,5}$
}

Received: 10 October 2016 / Accepted: 25 October 2016/Published online: 4 November 2016

(C) Springer-Verlag Berlin Heidelberg 2016

\begin{abstract}
Chronic myeloid leukaemia (CML) is consistently associated with the $B C R-A B L 1$ fusion gene located on the derivative chromosome 22 (Philadelphia chromosome, $\mathrm{Ph}+$ ve) as a result of a $\mathrm{t}(9 ; 22)(\mathrm{q} 34 ; \mathrm{q} 11.2)$ translocation. We describe a 36-year-old male in blast phase CML presenting with acute myelomonocytic leukaemia and eosinophilia. Five months after initial disease diagnosis and despite being treated with standard imatinib therapy (400 mg daily) conventional cytogenetic analysis (CCA) demonstrated the evolution of a chromosome 16 pericentric inversion (inv(16)(p13q22)) within the already $\mathrm{Ph}+$ ve cells. Bone marrow aspiration and biopsy revealed $60 \%$ blasts, positive for CD34, HLADR, CD33 and CD13. Fluorescence in situ hybridization (FISH) and reverse transcription-polymerase chain reaction (RT-
\end{abstract}

M. A. Catherwood and P. McGrattan contributed equally to the study.

Mark A. Catherwood

mark.catherwood@belfasttrust.hscni.net

Peter McGrattan

peter.mcgrattan@belfasttrust.hscni.net

1 Clinical Haematology, Belfast City Hospital, Belfast Health and Social Care Trust, Lisburn Road, Belfast, Northern Ireland BT9 7AB, UK

2 Northern Ireland Regional Genetics Service, Belfast City Hospital, Belfast Health and Social Care Trust, Lisburn Road, Belfast, Northern Ireland BT9 7AB, UK

3 Department of Experimental, Diagnostic and Specialty Medicine, Hematology/Oncology "L. e A. Seràgnoli”, University of Bologna, Bologna, Italy

4 Centre for Cancer Research and Cell Biology (CCRCB), Queen's University Belfast, Belfast, Northern Ireland, UK

5 Centre for Medical Education (CME), Queen's University Belfast, Belfast, Northern Ireland, UK
PCR) confirmed presence of a $C B F \beta-M H Y 11$ rearrangement whilst next generation sequencing (NGS) detected an E255K point mutation within the $B C R-A B L 1$ tyrosine kinase domain. Neither the $C B F \beta-M H Y 11$ rearrangement nor the E225K point mutation were present at diagnosis. The patient received a combination of daunorubicin $\left(60 \mathrm{mg} / \mathrm{m}^{2}\right)$ and cytarabine $\left(100 \mathrm{mg} / \mathrm{m}^{2}\right)$ and had his tyrosine kinase inhibitor (TKI) changed to nilotinib before undergoing a male unrelated donor (MUD) stem cell transplant (SCT). The $\mathrm{t}(9 ; 22)$ and inv(16) rearrangements persisted at day 100 post MUD SCT and his TKI was further switched to dasatinib. The patient presented with pleural effusions and had subsequent CNS relapse and passed away from relapsed disease and infective complications, 19-month post MUD allograft.

Keywords Chronic myeloid leukaemia $\cdot$ Blast phase · $\operatorname{Inv}(16)(\mathrm{p} 13 \mathrm{q} 22) / \mathrm{CBF} \beta-\mathrm{MHY} 11 \cdot$ Next generation sequencing · Conventional cytogenetic analysis · Imatinib . Nilotinib · Dasatinib

\section{Introduction}

Chronic myeloid leukaemia (CML) is a clonal myeloproliferative neoplasm characterized by the presence of the $\mathrm{t}(9 ; 22)(\mathrm{q} 34 ; \mathrm{q} 11.2)$ also known as the Philadelphia (Ph) chromosome which results in the formation of the $B C R-A B L 1$ fusion gene. $B C R-A B L 1$ protein has constitutive tyrosine kinase activity and plays a pivotal role in the pathogenesis of CML [1]. At diagnosis, $90-95 \%$ of cases of CML have the typical $t(9 ; 22)$ rearrangement while the remaining cases either have variant translocations involving a third or fourth chromosome (3- or 4-way reciprocal translocation variants), or have a cryptic translocation of $9 \mathrm{q} 34(\mathrm{ABL})$ and $22 \mathrm{q} 11.2$ (BCR) that can only be detected by fluorescence in situ 
hybridization (FISH), and reverse transcription-polymerase chain reaction (RT-PCR) [2].

Tyrosine kinase inhibitors (TKIs) targeting the BCR-ABL1 oncoprotein are the standard therapy for patients with CML. However, it has been estimated that $20-40 \%$ of patients receiving first-line imatinib therapy will eventually require an alternative treatment because of imatinib intolerance or resistance [3]. The best characterized mechanism of resistance is point mutations within the $B C R-A B L 1$ kinase domain that impair or prevent TKI binding [4].

Depending on the clinical setting (primary or secondary resistance and chronic or advanced phase), kinase domain mutations have been detected in $30-90 \%$ of patients with imatinib resistance and in $20-80 \%$ of patients who failed to respond to nilotinib or dasatinib therapy [4].
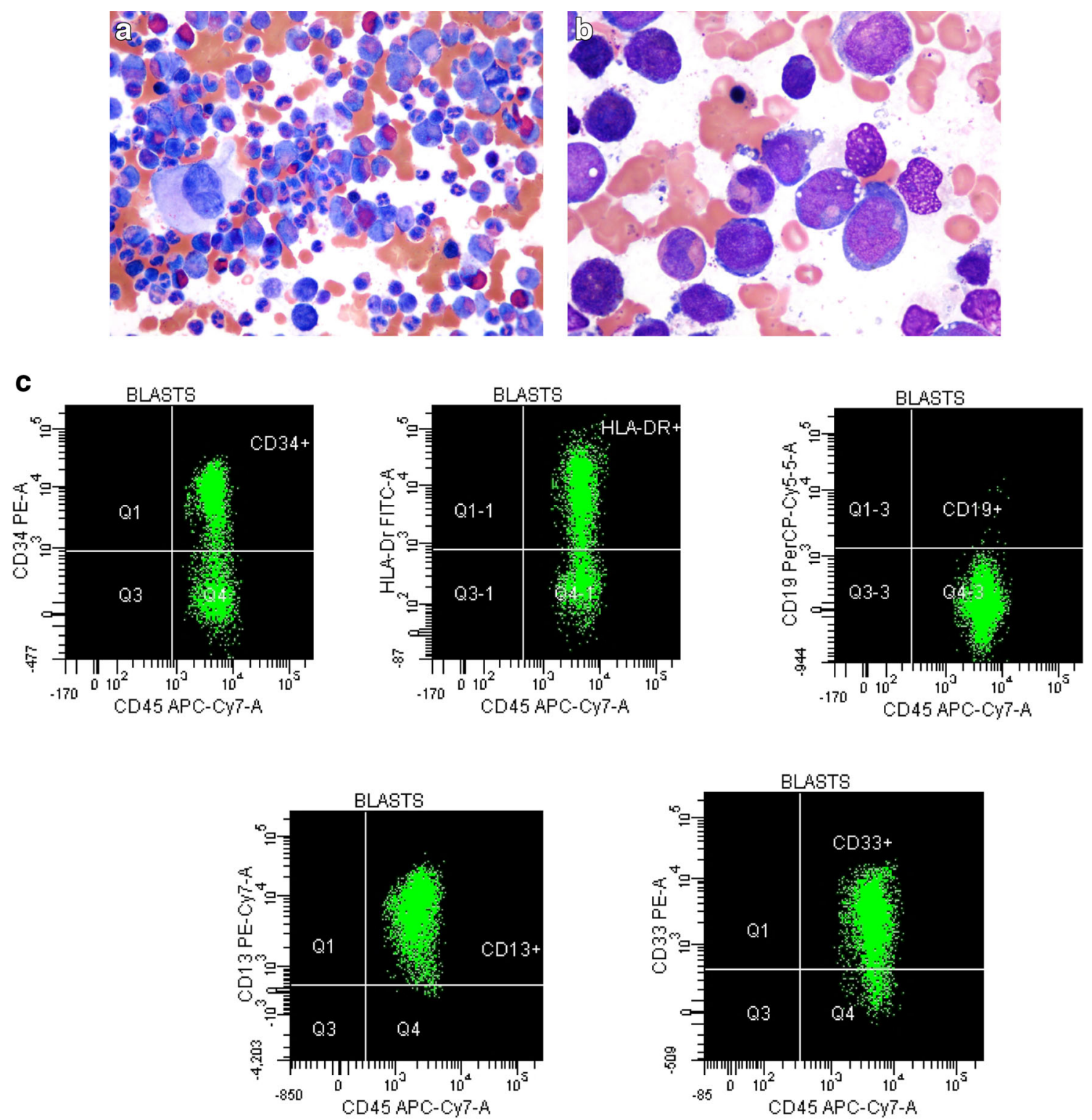

Fig. 1 a BM at diagnosis. b BM illustrating CML in blast crisis showing hypercellular bone marrow with $60 \%$ myeloid blasts and $5 \%$ eosinophils. $\mathbf{c}$ PB flow cytometry showing immunophenotyping data 
of de novo acute myeloid leukaemia (AML) such as the $\mathrm{t}(8 ; 21)(\mathrm{q} 21 ; \mathrm{q} 21), \mathrm{t}(15 ; 17)(\mathrm{q} 24 ; \mathrm{q} 21)$ and the $\operatorname{inv}(16)(\mathrm{p} 13 \mathrm{q} 22) / \mathrm{t}(16 ; 16)(\mathrm{p} 13 ; \mathrm{q} 22)$ have been described in CML-BC [7-9].

AML with inv(16) has been defined as a distinctive morphological subtype by French-American-British classification and is designated M4Eo [10]. The World Health Organization classification has since included in AML with recurrent cytogenetic abnormalities AML with $\operatorname{inv}(16)(\mathrm{p} 13.1 \mathrm{q} 22)$ or $\mathrm{t}(16 ; 16)(\mathrm{p} 13.1 ; \mathrm{q} 22) ; C B F B-M Y H 11$ in which there is characteristic abnormal eosinophil morphology [11]. The core-binding factor $\beta(C B F \beta)$ gene at
$16 \mathrm{q} 22$ and the myosin heavy chain gene (MYH11) at $16 \mathrm{p} 13$ are both disrupted and recombined to create a novel fusion gene, $C B F \beta-M Y H 11$ [12]. This fusion gene can be detected by conventional cytogenetics, FISH and RTPCR. Unlike de novo AML, inv(16)(p13q22) in CML-BC is not associated with a favourable prognosis [13].

We describe a CML patient in $\mathrm{BC}$ at which time the blasts had morphologic features of myelomonocytic leukaemia with excess eosinophils. Conventional cytogenetics showed inv(16)(p13q22) and FISH and RT-PCR proved the presence of $C B F \beta-M H Y 11$ which was absent at diagnosis.

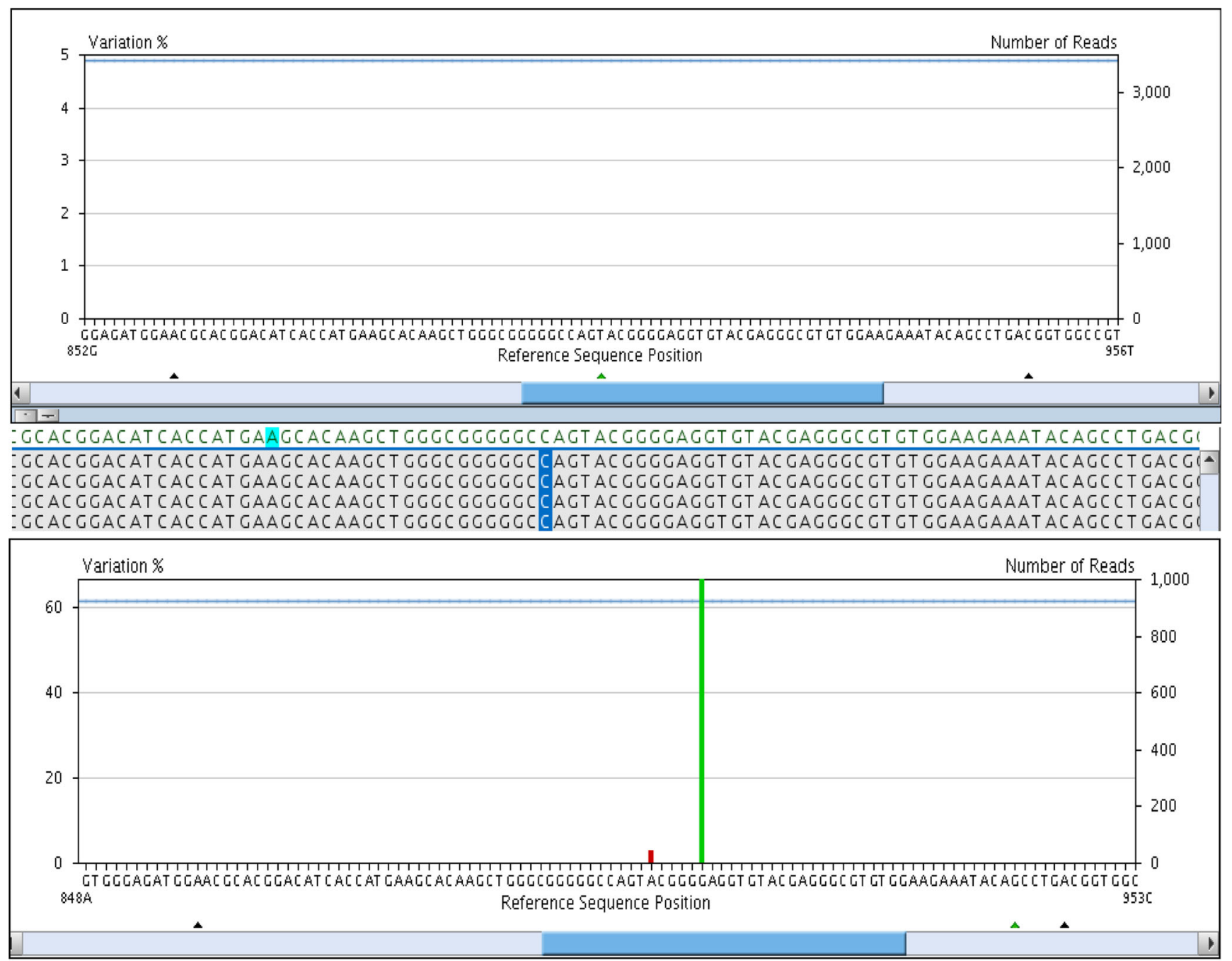

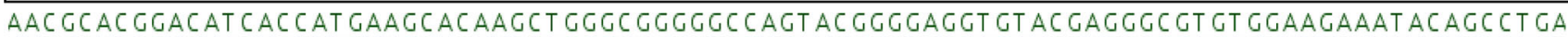

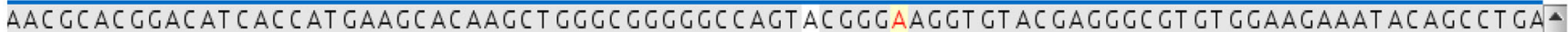
$A A C G C A C G G A C A T C A C C A T G A A G C A C A A G C T G G G C G G G G G C C A G T A C G G G A A G G T G T A C G A G G G C G T G T G G A A G A A A T A C A C C C T G A$ AAC GCACGGACAT C AC C AT GAAGCACAACCT GGCCGGGGCCCAGTACGCGGACGT CTAC GAGGCC CT GT GCAACAAATACACC CT GA

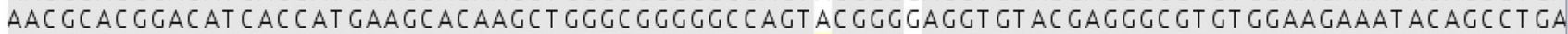

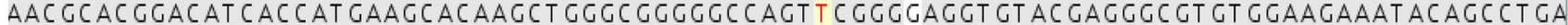
AACGCACGGACATCACCATGAAGCACAAGCT GGGCGGGGGCCAGTT CGGGGAGGT GTACGAGGGCGTGTGGAAGAAATACAGCCT GA

Fig. 2 Deep sequencing of the $B C R-A B L 1$ kinase domain-screenshots showing a portion of the global alignment of sequence reads to the reference $A B L 1$ sequence obtained with AVA software (Roche Applied Science) around codons 253-255. Upper panel: sample at diagnosis, no evidence of nucleotide substitutions were detected (sequencing depth, 3432 reads as shown on the right $Y$ axis). Lower panel: relapse sample showing an E255K (88.32\%; green bar) and a Y253F (3.28\%; red bar) kinase domain mutation 


\section{Case report}

A 36-year-old male patient presented with a 3-month history of weight loss, drenching night sweats and left upper quadrant pain. On examination he was noted to have splenomegaly with haemoglobin $77 \mathrm{~g} / \mathrm{L}$; white cell count (WCC) $366 \times 10^{9} / \mathrm{L}$ and a platelet count of $988 \times 10^{9} / \mathrm{L}$.

Bone marrow (BM) biopsy revealed a hypercellular marrow with myeloid hyperplasia, increased megakaryocytes and suppression of erythropoiesis but no excess blasts (Fig. 1). RTPCR on PB was positive for the p210 BCR-ABL1 transcript. BM chromosome analysis revealed a male $46, \operatorname{XYt}(9 ; 22)(\mathrm{q} 34 ; \mathrm{q} 11)$ karyotype in all ten metaphases examined. FISH analysis demonstrated a typical $B C R-A B L 1$ rearrangement in $99.2 \%$ of the $250 \mathrm{BM}$ nuclei examined.

A diagnosis of CML in chronic phase was established and he was treated with standard dose imatinib (400 mg daily) with normalization of counts. Five months later, his WCC was $115 \times 10^{9} / \mathrm{L}$. His blood film revealed leucoerythroblastic changes with a large number of circulating blasts. Upon admission to hospital, he deteriorated quite rapidly, developed pneumonia, type I respiratory failure and sustained a splenic infarct. He was admitted to intensive care for stabilisation and underwent embolisation of a splenic artery pseudoaneurysm.

BM biopsy and aspirate confirmed myeloid blast crisis with a hypercellular aspirate containing approximately $60 \%$ blasts and 5\% eosinophils (Fig. 2). Flow cytometry detected a population of myeloblasts (60\%), positive for CD34, HLADR, CD33 and CD13. BM chromosome analysis showed a male karyotype with the $t(9 ; 22)$ Philadelphia rearrangement previously detected at diagnosis and a pericentric inversion of chromosome 16 (p13p22) (inv(16)) in all ten metaphases examined. FISH/RT-PCR analysis confirmed a CBF $\beta-M H Y 11$ rearrangement. Next generation sequencing (NGS) [14] detected an E255K point mutation within the $B C R-A B L 1$ tyrosine kinase domain. Neither the CBF $\beta$ MHY11 rearrangement nor the E225K point mutation were present at disease diagnosis.

Standard AML induction chemotherapy DA3 +10 (daunorubicin $60 \mathrm{mg} / \mathrm{m}^{2}$ and cytarabine $100 \mathrm{mg} / \mathrm{m}^{2}$ ) was started and his TKI therapy was switched to nilotinib (400 mg bd daily). He was also tissue-typed and referred for a matched-unrelated bone marrow transplant as he had no sibling donors.

$\mathrm{He}$ recovered his blood counts suggesting transient remission however, for 7 weeks post-DA3 +10 induction chemotherapy; he relapsed again with blast crisis. He received salvage FLAG chemotherapy (fludarabine, cytarabine and idarubicin). A post FLAG chemotherapy BM biopsy revealed morphological remission chromosome analysis still showed persistence of the $t(9 ; 22)$ and inv(16) rearrangements $46, X Y, t(9 ; 22)(q 34 ; q 11), \operatorname{inv}(16)(p 13 q 22)$ with ten cells having both $t(9 ; 22)$ and inv(16) rearrangements, therefore he was not in remission.

Pre-SCT cytogenetic analysis done immediately on recovery of counts from induction showed the diagnostic $\mathrm{t}(9 ; 22)$ rearrangement in $83.3 \%$ ( 25 cells) of the 30 metaphases examined. Three $(12 \%)$ of these $\mathrm{Ph}+$ ve cells also showed the inv(16) rearrangement 46,XY,t $(9 ; 22)(\mathrm{q} 34 ; \mathrm{q} 11$, 22]/46idem,inv(16)(p13q22, 3]/46,XY \{5]. The marrow was relatively cellular with $2 \%$ blasts, $9 \%$ myelocytes,
Table 1 Summary of reported cases of inv(16) in CML in blast crisis

\begin{tabular}{lllll}
\hline Reference & Age/gender & Time to BC & Treatment & Survival \\
\hline Wu et al. 2006 [7] & 33, M & NA & Allo-BMT & Died 2 failed allo-BMT \\
Wu et al. 2006 [7] & 41, M & 3 & Allo-BMT & Alive 4 years post allo-BMT \\
Wu et al. 2006 [7] & 62, F & 4 & Imatinib & Died 24 months \\
Merzianu et al. 2005 [8] & 43, F & 11 & Imatinib & Died 3 months \\
Merzianu et al. 2005 [8] & $61, \mathrm{~F}$ & 0 & Hydroxyurea & Died 7 months \\
Merzianu et al. 2005 [8] & $47, \mathrm{M}$ & 8 & Interferon & Died 1 months \\
Merzianu et al. 2005 [8] & $36, \mathrm{~F}$ & 7 & Hydroxyurea & Died 1 months \\
Patel et al. 2006 [13] & 64, M & 6 & Allo-SCT & Died 16 months \\
Patel et al. 2006 [13] & 56, M & 0 & Hydroxyurea & Died 3 months \\
Tsuboi et al. 2002 [15] & $44, \mathrm{M}$ & 84 & Hydroxyurea & Died 3 months \\
Myint et al. 1997 [16] & 29, M & 3 & Hydroxyurea & Died 0 months \\
Evers et al. 1992 [17] & $39, \mathrm{M}$ & 3 & Hydroxyurea & Died 10 months \\
Asou et al. 1992 [18] & $51, \mathrm{M}$ & 1 & Hydroxyurea & Died 3 months \\
Enright et al. 1992 [19] & $78, \mathrm{M}$ & 4 & Hydroxyurea & Died 3 months \\
Heim et al. 1992 [20] & 21, M & 2 & Busulfan & Alive 48 months \\
Secker-Walker et al. [21] & 25, M & 0 & Allo-BMT & Died 15 months \\
Ninomiya et al. 2011 [22] & $63, \mathrm{~F}$ & 0 & Imatinib & Died 7 months \\
\hline
\end{tabular}

$M$ male, $F$ female, $B C$ blast crisis, $N A$ not available, $B M T$ bone marrow transplantation, Allo-SCT allogeneic stem cell transplantation 
$13 \%$ promyelocytes but no eosinophils or basophils and consistent with chronic phase CML. He proceeded directly to a myeloablative MUD SCT, with cyclophosphamide and total body irradiation (TBI) conditioning. He engrafted by day 21. Post-transplant he suffered from severe mucositis. He also required foscarnet for cytomegalovirus (CMV) reactivation. He developed Grade I cutaneous graft versus host disease. The treatment plan was to restart his nilotinib therapy post SCT but this was delayed due to deranged liver function tests (LFT).

On day 100 post SCT he relapsed again, the $t(9 ; 22)$ and inv(16) rearrangements detected at CML-BC were again present. His tyrosine kinase inhibitor was switched from nilotinib to dasatinib (100/150 mg alternate days). He was treated with mitoxantrone to control his white cell count and then received two donor lymphocyte infusions (DLI) which has been successful in achieving a fourth haematological remission. A bone marrow aspirate at this stage revealed a hypocellular aspirate with no excess of blasts. Cytogenetics confirmed a complete remission with normal cytogenetics, karyotype 46,XY.

The patient presented with headaches and MRI and lumbar puncture confirmed CNS relapse of disease. The CNS relapse consisted of $37 \%$ blasts and $1 \%$ eosinophils. He received intrathecal chemotherapy (cytarabine, hydrocortisone and methotrexate), but required cranial irradiation to eradicate disease. BM and BCR-ABL/ABL testing confirmed ongoing morphological and molecular remission, respectively. Dasatinib TKI was increased to $100 \mathrm{mg} / 150 \mathrm{mg}$ alternate days. During outpatient follow-up, low molecular evidence ( $B C R-A B L / A B L 0.08 \%$ ) of relapse on two consecutive tests were noted. Three months later, the patient, presented as an emergency with shortness of breath, was found to have not only pleural effusions, but haematological evidence of disease relapse in blast crisis with mainly blasts in his blood. He had subsequent CNS relapse, and passed away shortly after this from infective complications (Staphylococcus aureus bacteraemia) 19 months post MUD allograft.

\section{Discussion}

The cellular mechanism which drives the transition of CML-CP to CML-BC is currently unknown. However, it is well recognized that during this transition common additional abnormalities in CML-BC lead to a gain or loss of genetic material such as extra copies of $\mathrm{Ph}$, trisomy 8 , isochromosome $17 \mathrm{q}$, or trisomy 19 inferring these additional hits are in part responsible for the transition [6]. The coexistence of $\operatorname{inv}(16)$ and $t(9 ; 22)$ is rare with a limited number of reports in the literature over the past 25 years (Table 1 ). These cases have both been described in blast crisis of CML and also de novo AML. Most of the de novo forms of AML with the $t(9 ; 22)$ and inv(16) have a favourable prognosis. CML-BC with these abnormalities are typically characterized by an aggressive clinical course with rapid disease progression and resistance to treatment $[7,8,13]$. In common with this case, most have shown a sudden onset with only several months from first presentation to CML-BC (Table 1).

It is well recognized that the presence of $B C R-A B L 1$ kinase domain point mutations are suggestive of genetic instability and of increased risk of progression and are detectable in up to $50 \%$ of patients with treatment failure and progression [4]. In our case, imatinib was only effective for 5 months for the control of chronic phase before transformation. At $\mathrm{BC}$, we detected the presence of $C B F \beta-M H Y 11$ and $\mathrm{E} 255 \mathrm{~K}$ point mutation within the $B C R-A B L 1$ tyrosine kinase domain. To investigate, this further we used NGS (22) comparing diagnostic and BC samples. Of interest, the $\mathrm{BC}$ sample consisted of an E255K clone representing $90.5 \%$ and a smaller clone consisting of Y253F (3.69\%) which would be below the threshold of conventional Sanger sequencing. However, the diagnostic sample did not contain either mutated clone at a sensitivity of $1 \%$ over 4000 reads. This sample also did not contain the $C B F \beta-M H Y 11$ transcript.

The E255K mutation occurs within the P-loop of the kinase domain and is a common feature in imatinib resistance cases and is well recognized to have increased oncogenic potency despite reduced kinase activity compared with unmutated BCR-ABL.

Our patient survived 23 months from his presentation in $\mathrm{BC}$. This is at the longer end of the survival spectrum in the case series but despite bone marrow transplant, DLis and TKIs he did not achieve anymore prolonged survival.

In summary, although the presence of inv(16) is a rare occurrence in CML-BC, this is still associated with a poor prognosis even in the TKI era. The vast majority of patients have succumbed to their disease and highlights the need for further treatment options in this group of patients and the value of continued monitoring for disease progression.

\section{Compliance with ethical standards}

Conflict of interest The authors declare that they have no conflict of interest.

Ethical approval All procedures performed in studies involving human participants were in accordance with the ethical standards of the institutional and/or national research committee and with the 1964 Helsinki declaration and its later amendments or comparable ethical standards.

Informed consent Informed consent was obtained from all individual participants included in the study. 


\section{References}

1. Vardiman JW, Melo JV, Baccarani M, Thiele J (2008) Chronic myelogenous leukaemia, $B C R-A B L$ positive. In: Swerdlow $\mathrm{SH}$, Campo E, Harris NL, Jaffe ES, Stein H, Thiele J, Vardiman JW (eds) WHO classification of tumours of haematopoietic and lymphoid tissues. IARC, Lyon, pp. 32-37

2. Melo JV (1996) The diversity of BCR-ABL fusion proteins and their relationship to leukemia phenotype. Blood 88:2375-2384

3. Milojkovic D, Apperley J (2009) Mechanisms of resistance to imatinib and second-generation tyrosine inhibitors in chronic myeloid leukemia. Clin Cancer Res 15:7519-7525

4. Soverini S, Hochhaus A, Nicolini FE et al (2011) BCR-ABL kinase domain mutation analysis in chronic myeloid leukemia patients treated with tyrosine kinase inhibitors: recommendations from an expert panel on behalf of European LeukemiaNet. Blood 118(5): $1208-1215$

5. Sacchi S, Kantarjian H, O’Brien S et al (1999) Chronic myelogenous leukemia in nonlymphoid blastic phase: analysis of the results of first salvage therapy with three different treatment approaches for 162 patients. Cancer 86:2632-2641

6. Ruff P, Saragas E, Poulos M, Weaving A (1995) Patterns of clonal evolution in transformed chronic myelogenous leukemia. Cancer Genet Cytogenet 81:182-184

7. Wu T, Slovak ML, Synder DS, Arber DA (2006) Coexistence of inversion 16 and the Philadelphia chromosome in acute and chronic myeloid leukemias: report of six cases and review of the literature. Am J Clin Pathol 125:260-266

8. Merzianu M, Medeiros LJ, Cortes J et al (2005) inv(16)(p13q22) in chronic myelogenous leukemia in blast phase: a clinicopathologic, cytogenetic, and molecular study of five cases. Am J Clin Pathol 124:807-814

9. Johansson B, Fioretos T, Mitelman F Cytogenetic and molecular genetic evolution of chronic myeloid leukemia. Acta Haematol 107:76-94

10. Bennett JM, Catovsky D, Daniel MT et al (1985) Proposed revised criteria for the classification of acute myeloid leukemia: a report of the French-American-British Cooperative Group. Ann Intern Med 103:620-625

11. Arber DA, Brunning RD, Le Beau MM et al (2008) Acute myeloid leukemia with recurrent cytogenetic abnormalities. In: Swerdlow $\mathrm{SH}$, Campo E, Harris NL et al (eds) WHO classification of tumours of haematopoietic and lymphoid tissues. IARC Press, Lyon, pp. 111-112 World Health Organization Classification of Tumours

12. Liu P, Tarle SA, Hajra A et al (1993) Fusion between transcription factor CBF beta/PEBP2 beta and a myosin heavy chain in acute myeloid leukemia. Science 261:1041-1044

13. Patel BB, Mohamed AN, Schiffer CA (2006) Acute myelogenous leukemia like translocations in CML blast crisis: two new cases of $\operatorname{inv}(16) / t(16 ; 16)$ and a review of the literature. Leu Res 30:225-232

14. Soverini S, De Benedittis C, Machova Polakova K et al (2013) Unraveling the complexity of tyrosine kinase inhibitor-resistant populations by ultra-deep sequencing of the BCR-ABL kinase domain. Blood 122(9):1634-1648

15. Tsubol K, Komatsu H, Miwa H et al (2002) Lymphoid blast crisis of chronic myelogenous leukaemia with inv(16)(p13;q22). Leuk Res 26:771-774

16. Myint H, Ross FM, Hall JL, Hamblin TJ (1997) Early transformation to acute myeloblastic leukaemia with the acquisition of inv(16) in $\mathrm{Ph}$ positive chronic granulocytic leukaemia. Leuk Res 21:473-474

17. Evers JP, Bagg A, Himoe E et al (1992) Temporal association of marrow eosinophilia with inversion of chromosome 16 in recurrent blast crisis of chronic myelogenous leukemia. Cancer Genet Cytogenet 62:134-139

18. Asou N, Sanada I, Tanaka K et al (1992) Inversion of chromosome 16 and bone marrow eosinophilia in a myelomonocytic transformation of chronic myeloid leukemia. Cancer Genet Cytogenet 61:197-200

19. Enright H, Weisdorf D, Peterson L et al (1992) Inversion of chromosome 16 and dysplastic eosinophils in accelerated phase of chronic myeloid leukemia. Leukemia 6:381-384

20. Heim S, Christensen BE, Fioretos T et al (1992) Acute myelomonocytic leukemia with inv (16)(p13q22) complicating Philadelphia chromosome positive chronic myeloid leukemia. Cancer Genet Cytogenet 59:35-38

21. Secker-Walker LM, Morgan GJ, Min T et al (1992) Inversion of chromosome 16 with the Philadelphia chromosome in acute myelomonocytic leukemia with eosinophilia: report of two cases. Cancer Genet Cytogenet 58:29-34

22. Ninomiya S, Kanemura N, Tsurumi $\mathrm{H}$ et al (2011) Coexistence of inversion 16 and the Philadelphia chromosome comprising P190 $\mathrm{BCR} / \mathrm{ABL}$ in chronic myeloid leukemia blast crisis. In J Hematol 93:806-810 